\title{
Onabotulinum toxin A for treatment of chronic migraine with medication overuse
}

\author{
L. Grazzi
}

(c) European Union 2013

\begin{abstract}
Chronic migraine is a common and debilitating headache syndrome. Botulinum neurotoxin (BoNT), a potent toxin produced by the anaerobic bacterium Clostridium botulinum, used largely for treatment of disorders associated with increased muscle tone and hyperidrosis, has been recently used for patients suffering from chronic migraine. In this study, two groups of patients were treated with different dosages of BoNT A to verify the efficacy for chronic migraine. The results confirmed the efficacy of BoNT A when used at the dosage of $150 \mathrm{U}$. In the second group of patients treated with $100 \mathrm{U}$, results were not significant. Although these results are preliminary, they led to intense efforts to evaluate the analgesic properties of BoNT A and to assess their clinical applicability.
\end{abstract}

Keywords Onabotulinum toxin A - Chronic migraine . Medication overuse

\section{Introduction}

Chronic migraine is a common and debilitating headache syndrome and it affects $1.4-2.2 \%$ of the general population [1]. These patients experience headache $>15$ days per month for $>3$ months, and often they overuse medications for aborting pain for more that 15 tablets per months (generally triptans or NSAIDS).

L. Grazzi $(\square)$

Department of Clinical Neuroscience, Headache Unit,

C. Besta Neurological Institute and Foundation,

Via Celoria, 11 20133, Milan, Italy

e-mail: grazzi.1@istituto-besta.it
An effective prophylactic treatment, often after an adequate withdrawal from offending medications, improves the clinical condition of these patients by reducing the medication consumption [2].

Onabotulinum toxin A has been reported to improve pain in several conditions including migraine [3-5]. Various onabotulinum toxin A dosages and injection paradigms have been evaluated in several recent studies and the PREEMPT protocol evolved from these preceding paradigms, by showing definitely that onabotulinum toxin A is a safe, well-tolerated and effective headache prophylactic treatment for chronic migraine [6].

Botulinum neurotoxin a potent toxin produced by the anaerobic bacterium Clostridium botulinum, used largely for treatment of disorders associated with increased muscle tone and hyperidrosis, has been recently used for patients suffering from chronic migraine [6].

The mechanism of onabotulinum toxin A in antinociception has not been clearly clarified yet: probably, as demonstrated in some studies, onabotulinum toxin A inhibits the release of nociceptive mediators (glutamate, substanceP, CGRP) from peripheral terminals of primary afferents [7, 8].

Blocking the release of these neurotransmitters inhibits neurogenic inflammation and consequently the peripheral sensitization of nociceptive nerve fibers [7-9].

As the significant population of chronic migraine patients, refractory to common therapeutic prophylaxis, onabotulinum toxin A can be considered a new option for these problematic patients. Onabotulinum toxin A has been used in our clinical experience for treating patients referring to our headache centre and suffering from chronic migraine with medication overuse.

Aim of this study was to evaluate two groups of patients treated with different dosages of BoNT A to verify the efficacy for chronic migraine. 


\section{Method}

Two groups of patients suffering from chronic migraine with medication overuse (diagnosis made according with the IHS criteria 2004 revised in 2006) [10-11] were studied.

Patients were treated by a withdrawal protocol in a day hospital regimen for 5 days to stop the overuse of symptomatic medications. After that, patients were treated by onabotulinum toxin A according with different schedule of treatment.

In the first group, ten patients have been treated by Onabotulinum toxin A injection in multiple sites according to the protocol of the PREEMPT study [6] at the dosage of 150 UI for 31 sites. Every session of local injection (150 UI per 31 sites) has been repeated every 3 months for a period of almost 1 year. A second group of 8 patients were treated by onabotulinum toxin A 100 UI, injection in multiple sites according to their clinical condition. In this last phase of the study, patients had to pay for the treatment a fee for every session. For these patients too, every session of local injection was repeated every 3 months for a period of almost 1 year. Clinical indexes were recorded using a headache daily diary, with the number of medication intake per month and the days of headache per month.

Patients were followed for 1 year.

\section{Results}

In the first group of patients, treated with 150 UI, days of headache/month decreased during the period of treatment at $150 \mathrm{UI}$ (pre $21.4 \pm 7.9$ post $13.8 \pm 10.9 p<0.01$ ). Also medication intake decreased (pre $19.6 \pm 8$ post $11.7 \pm 9.5 p<0.01)$.

In the second group of patients, treated with $100 \mathrm{UI}$, results were not significant: only 3 patients completed the treatment with 4 sessions and they did not reported any advantage; 5 patients completed only the first session of treatment schedule and they stopped treatment for different reasons: 3 patients referred they did not have any change in clinical condition and they decided that the treatment was too expensive; 2 patients interrupted the treatment as they reported ptosis after injection.

\section{Discussion}

Although these results are very preliminary, they led to intense efforts to evaluate analgesic properties of onabotulinum toxin A and to assess their clinical applicability.
The pharmacological profile of onabotulinum toxin A makes it a good candidate for migraine prevention at the adequate dosage as proposed in the PREEMPT study. Its long duration of action (3 months) makes it particularly attractive for patients who are not compliant with the daily use of preventive medications, or if they cannot tolerate it or when they are refractory to preventive medications.

On the other side, as result of several factors (not excluded the problem concerning that the treatment was too expensive) we cannot confirm the efficacy of lower dosage (100 UI) for patients with chronic migraine, submitted to the treatment with onabotulinum toxin A after withdrawal.

Conflict of interest The author certifies that there is no actual or potential conflict of interest in relation to this article.

\section{References}

1. Diener HC, Limmroth V (2004) Medication-overuse headache: a worldwide problem. Lancet Neurol 3:475-483

2. Grazzi L, Andrasik F, Usai S, Bussone G (2009) Treatment of chronic migraine with medication overuse: is drug withdrawal crucial? Neurol Sci 30(S1):85-88

3. Ranoux D, Attal N, Morain F, Bouhasira D (2008) Botulinum toxin type A induces direct analgesic effects in chronic neuropathic pain. Ann Neurol 2008(64):274-283

4. Mathew N, Frishberg BM, Gawel M, Dimitrova R, Gibson J, Turkel C (2005) Botulinum toxin type A (BOTOX) for the prophylactic treatment of chronic daily headache. A randomized, double blind, placebo controlled trial. Headache 45:293-307

5. Sandrini G, Perrotta A, Tassorelli C, Torelli P, Brighina F, Sances G, Nappi G (2011) Botulinum toxin type-A in the prophylaxis treatment of medication-overuse headache: a multi center, double blind, randomized, placebo-controlled, parallel group study. J Head pain 12:427-433

6. Blumenfeld A, Silberstein S, Dodick D, Aurora S, Turkel C, Binder W (2010) Method of injection of Onabotulinum toxin A for chronic migraine: a safe, well-tolerated and effective treatment paradigm based on the PREEMPT clinical program. Headache 50:1406-1418

7. Aoki KR (2005) Review of a proposed mechanisms for the antinociceptive action of botulinum toxin type A. Neurotoxicology 26:785-793

8. Aoki KR (2003) Evidence for antinociceptive activity of botulinum toxin type A in pain management. Headache 43(suppl):S9S15

9. Gazerani P, Pedersen NS, Staahl C, Drewes AM, Arendt-Nielsen L (2009) Subcutaneous botulinum toxin type A reduces capsaicin-induced trigeminal pain and vasomotor reactions in human skin. Pain 141:60-69

10. Headache Classification Subcommittee of the International Headache Society (2004) The International classification of headache disorders-2nd edition. Cephalalgia 24(suppl 1):1-160

11. Olesen J, Bousser MG, Diener HC, Dodick D, First M, Goadsby PJ, Goebel H, Lainez MJA, Lance JW, Lipton RB, Nappi G, Sakai F, Schoenen J, Silberstein SD, Steiner TJ (2006) New appendix criteria open for a broader concept of chronic migraine. Cephalalgia 26:742-746 